\title{
Instability and control of a periodically driven Bose-Einstein condensate
}

\author{
C. E. Creffield \\ Departamento de Física de Materiales, Universidad Complutense de Madrid, E-28040 Madrid, Spain
}

(Received 10 September 2008; published 5 June 2009)

\begin{abstract}
We investigate the dynamics of a Bose-Einstein condensate held in an optical lattice under the influence of a strong periodic driving potential. Studying the mean-field version of the Bose-Hubbard model reveals that the condensate becomes dynamically unstable when the effective intersite tunneling becomes negative. We further show how controlling the sign of the tunneling can be used to manage the dispersion of an atomic wave packet.

DOI: $10.1103 /$ PhysRevA.79.063612

PACS number(s): 67.85.- d, 03.65.Xp, 03.75.Kk, 03.75.Lm
\end{abstract}

\section{INTRODUCTION}

The spectacular experimental progress in confining BoseEinstein condensates in optical lattice potentials has provided a powerful tool for investigating many-body quantum dynamics. Such optical potentials are extremely clean and controllable, and together with their long decoherence times, this allows the observation of many coherent lattice phenomena which are highly challenging to study in solid-state systems. One such effect is "coherent destruction of tunneling" (CDT) [1] in which a periodic driving field acts to renormalize the tunneling between lattice sites. Although first noted as a single-particle effect [2], CDT also occurs in interacting systems such as atomic condensates [3] and has been observed extremely recently $[4,5]$ in cold atom experiments. The control that CDT provides over the condensate dynamics is achieved without altering any of the parameters of the optical lattice and so has natural applications to quantum information [6] since it preserves the system's coherence. However, it is crucial to know if the condensate remains stable during its time evolution and in particular whether dynamical instability occurs, in which deviations from a steady state grow exponentially with time. The case of a static potential was analyzed in Ref. [7] and studied experimentally in Refs. $[8,9]$, and it was found that dynamical instability occurs above a certain critical quasimomentum. Investigations of uniformly accelerated lattices $[10,11]$ also revealed the presence of dynamical instability, finding the nonintuitive result that this was enhanced in the limit of low acceleration.

In this paper we analyze the richer and more complex case where the condensate is periodically driven. To achieve this we follow the strategy in Ref. [11] and carry out the stability analysis about the Floquet states [1] of the system, which are the appropriate generalization of energy eigenstates to the case of a time-periodic Hamiltonian. An important point for experiment is to minimize or avoid instabilities, and so we first find the critical interaction strength at which dynamical instability occurs. We then connect this with the behavior of the effective tunneling parameter in the driven system, $J_{\text {eff }}$, and show how CDT can be used to manipulate $J_{\text {eff }}$ in order to provide control over the nonlinear matter-wave dispersion [12].

\section{METHOD}

Interactions between ultracold bosons are typically well described by a very short-range pseudopotential, param- etrized by the $s$-wave scattering length. If the interaction energies in the system are smaller than the excitation energy to the first excited Bloch band, then a system of bosons held in an optical lattice can be described very accurately by the Bose-Hubbard Hamiltonian [13]. For a one-dimensional system this is written as

$$
H_{\mathrm{BH}}=-J \sum_{\langle m, n\rangle}\left(a_{m}^{\dagger} a_{n}+\text { H.c. }\right)+\frac{U}{2} \sum_{m} n_{m}\left(n_{m}-1\right),
$$

where $a_{m}^{\dagger}\left(a_{m}\right)$ are the boson creation (annihilation) operators for a particle on lattice site $m, n_{m}=a_{m}^{\dagger} a_{m}$ is the a standard number operator, and the sites $\langle m, n\rangle$ are nearest neighbors. The properties of the system are then governed by the hopping parameter $J$ and the Hubbard interaction $U$ which describes the potential energy between two bosons occupying the same lattice site. An extremely valuable means of studying and controlling such systems is to accelerate the lattice by varying the phase difference between the two laser beams forming the standing wave potential. In the rest frame of the lattice this acceleration manifests itself as an inertial force which effectively "tilts" the potential, allowing, for example, investigation of Bloch oscillations [14]. If, however, instead of a uniform acceleration the lattice is periodically accelerated and decelerated, the potential will instead oscillate periodically in time, $H_{I}=K \cos (\omega t) \Sigma_{m} m n_{m}$, where $K$ and $\omega$ parametrize its amplitude and frequency, respectively.

In order to study the stability of the driven condensate we will first pass to a mean-field description of this model, analogous to the Gross-Pitaevskii equation, and then linearize about the ground state to obtain the Bogoliubov equations for the condensate excitations. This procedure will be valid as long as the number of atoms excited from the condensate is small compared to the number of condensed atoms. We first write the Heisenberg equations of motion for the boson operators $a_{n}$ and then take the classical field approximation and treat them simply as $c$ numbers $\alpha_{n}$. It is convenient [11] to use the scaling $\alpha_{n}=a_{n} / \sqrt{N}$, where $N$ is the average number of atoms per site. It is then straightforward to show that the classical amplitudes obey the equation of motion

$$
i \frac{\partial \alpha_{n}}{\partial t}=-J\left(\alpha_{n+1}+\alpha_{n-1}\right)+g\left|\alpha_{n}\right|^{2} \alpha_{n}+K \cos (\omega t) n \alpha_{n},
$$

where for simplicity we write the interaction as $g=N U$, and the amplitudes have the normalization $\Sigma_{j}\left|\alpha_{j}\right|^{2}=N_{\text {latt }}$, where $N_{\text {latt }}$ is the number of lattice sites. Note that we also take $\hbar$ 
$=1$ and will measure all energies in units of $J$.

To simplify the analysis we use periodic boundary conditions. In the limit of large lattice sizes, however, the choice of boundary conditions does not affect the underlying physics, and we will later use Dirichlet boundary conditions to simulate the time evolution of the condensate. In the absence of interactions, the eigenstates of the system will simply be plane waves $\alpha_{n}=\exp [$ inp], where $p$ is the wave's (quantized) momentum. With this in mind, we take as a trial solution $\alpha_{n}^{0}=\exp [i(n \phi+\theta)]$, where $\phi$ and $\theta$ are functions to be determined. Substituting this solution in Eq. (2) yields the results

$$
\begin{gathered}
\phi(t)=p-\frac{K}{\omega} \sin \omega t, \\
\theta(t)=2 J[\cos (p) S(t)-\sin (p) C(t)]-g t,
\end{gathered}
$$

where the functions $S(t) / C(t)$ are defined in terms of Bessel functions as

$$
\begin{gathered}
C(t)=\sum_{m=-\infty}^{\infty} \frac{\cos m \omega t-1}{m \omega} \mathcal{J}_{m}(K / \omega), \\
S(t)=\sum_{m=-\infty}^{\infty} \frac{\sin m \omega t}{m \omega} \mathcal{J}_{m}(K / \omega) .
\end{gathered}
$$

Note that in Eq. (3), $p$ is a constant of integration whose value is set by the requirement that in the absence of driving we recover standard plane waves as the eigenstates of the system.

Since the Hamiltonian of the system is periodic in time, the Floquet theorem dictates that the solutions of the timedependent Schrödinger equation can be written in the form $\exp [-i \epsilon t] \psi(t)$, where $\epsilon$ is termed the quasienergy and $\psi(t)$ is a $T$-periodic function called the Floquet state. To obtain the quasienergies we thus simply have to extract the terms from the solution which are not $T$ periodic, giving the result

$$
\epsilon(p)=-2 \cos (p) J \mathcal{J}_{0}(K / \omega)+g .
$$

In the absence of the driving, the quasienergies thus form a normal single-particle band structure, the interaction $g$ acting merely to shift the entire spectrum. The driving then acts to renormalize the width of the spectrum by the Bessel function $\mathcal{J}_{0}$, as was previously observed in a theoretical analysis [15] of semiconductor superlattice systems. In Fig. 1(a) we show numerical results for the quasienergies of an eight-site system, obtained directly from the time evolution of the system, which beautifully corroborate the expected behavior. In particular, when the Bessel function becomes zero, the spectrum collapses to a point and the system will manifest CDT.

In order to analyze the dynamical stability of the ground state $(p=0)$, we now introduce a perturbation $\alpha_{n}(t)$ $=\alpha_{n}^{0}(t)\left(1+u(t) \exp [i q n]+v^{*}(t) \exp [-i q n]\right)$, where $q$ is the momentum of the excitation relative to the condensate. We then linearize Eq. (2) about this solution to obtain the Bogoliubov-de Gennes equations for $u(t)$ and $v(t)$,

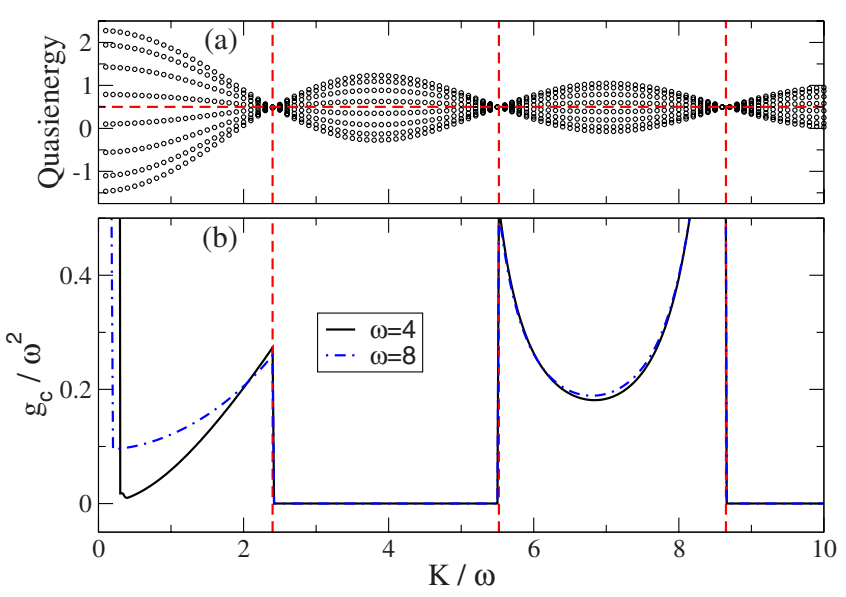

FIG. 1. (Color online) (a) Numerical results for the quasienergy spectrum of the driven mean-field Bose-Hubbard model for an eight-site system with $\omega=16$. The width of the spectrum is modulated by the Bessel function $\mathcal{J}_{0}(K / \omega)$ and displaced by the interaction energy $g=0.5$, in full agreement with the analytical solution [Eq. (7)]. (b) Plot of the critical interaction, $g_{c}$, at which the system becomes dynamically unstable when prepared in the $p=0$ state. The driving frequencies are $\omega=4$ (solid black line) and $\omega=8$ (dotteddashed blue line). The critical interaction diverges at $K / \omega=0$ and peaks at the Bessel function zeros (vertical dashed lines). When $J_{\text {eff }}$ is negative $g_{c}$ becomes zero, indicating that dynamical instability occurs for any positive value of $g$.

$$
i \frac{d}{d t}\left(\begin{array}{l}
u(t) \\
v(t)
\end{array}\right)=\mathcal{L}(q, t)\left(\begin{array}{l}
u(t) \\
v(t)
\end{array}\right),
$$

where the elements of the matrix $\mathcal{L}(q, t)$ are given by

$$
\begin{gathered}
\mathcal{L}_{11}(q, t)=4 J \sin (q / 2) \sin (q / 2-K / \omega \sin \omega t)+g, \\
\mathcal{L}_{12}(q, t)=g=-\mathcal{L}_{21}(q, t), \\
\mathcal{L}_{22}(q, t)=-4 J \sin (q / 2) \sin (q / 2+K / \omega \sin \omega t)-g .
\end{gathered}
$$

We can note that, similarly to the Hamiltonian, the operator $\mathcal{L}(q, t)$ is $T$ periodic. Consequently we can also apply the Floquet theorem to describe the time evolution of the quasiparticle excitation $(u, v)$. To find the corresponding Floquet states, we numerically evolve Eq. (8) over one period of driving, using the $2 \times 2$ identity matrix as the initial state. The result of this procedure is the single-period propagator $U$. The eigenstates of $U$ are then the excitation Floquet states, while its eigenvalues are related to the excitation quasienergies via $\lambda_{i}=\exp \left[-i T \epsilon_{i}\right]$.

The condition of dynamical stability can now be recast [16] in terms of the eigenvalues of $\mathcal{L}(q, t)$, in analogy with the use of Lyapunov exponents in classical mechanics. Instability is signaled by the quasienergies acquiring an imaginary component, corresponding to the exponential growth or decay of excitations. This in turn is revealed by the behavior of $\left|\lambda_{i}\right|$; if this is equal to 1 then the solution is stable, but if it exceeds 1 then instability occurs. The symmetries of $U$, combined with the normalization condition obeyed by the quasiparticle excitation, $|u|^{2}-|v|^{2}=1$, allow the characteristic equation to be written in the particularly simple form [11] 


$$
\lambda_{ \pm}=\operatorname{Re}\left[U_{11}\right] \pm \sqrt{\operatorname{Re}\left[U_{11}\right]^{2}-1} .
$$

From this expression it is clear that the stability of the excitation can be established by simply evaluating $\operatorname{Re}\left[U_{11}\right]$. If this quantity is less than 1 , then $\left|\lambda_{ \pm}\right|=1$ and the solution is stable. Conversely, finding $\operatorname{Re}\left[U_{11}\right]>1$ indicates instability. This suggests a simple scheme to map out the stability zones of the driven system. For a given choice of $K$ and $\omega$ we select a value of $g$ and scan over the range of $q$. If $\operatorname{Re}\left[U_{11}\right] \leq 1$ for all values of $q$ we can declare that the system is stable for these parameters and that to induce instability we need to increase $g$ to a higher value. In this way a standard bisection scheme can be used to locate the instability boundary, $g_{c}$.

\section{RESULTS}

We show the results of this procedure for two driving frequencies in Fig. 1(b). We can first observe that for $K / \omega$ $=0$ it is possible to directly diagonalize $\mathcal{L}(q)$ to obtain the result $\epsilon_{ \pm}= \pm 2 \sqrt{2} \sin (q / 2) \sqrt{2 J^{2} \sin ^{2}(q / 2)+J g}$. As expected, this duplicates the familiar result for the Bogoliubov excitations of an undriven stationary condensate [17]. It is also clear from this expression that dynamical instability will not occur if the interaction is repulsive since the eigenfrequencies will not become complex unless the product $(J g)<0$. Accordingly, as $K / \omega \rightarrow 0$ we can see from Fig. 1(b) that the value of $g_{c}$ diverges. On increasing $K / \omega$, the value of $g_{c}$ then rapidly drops, passing through a broad local minimum before again peaking as $K / \omega$ approaches 2.4048 - the first zero of $\mathcal{J}_{0}$. This corresponds to the onset of CDT; as the effective tunneling is reduced, the dynamics of the condensate is suppressed, and stability is regained.

Passing through the zero of $\mathcal{J}_{0}$, we can see that dynamical stability is then abruptly lost. In this region the condensate becomes dynamically unstable for any positive value of the interaction. We can obtain some insight into this effect from Eq. (7) by defining the effective tunneling, $J_{\text {eff }}=J \mathcal{J}_{0}(K / \omega)$. When $K / \omega$ is increased from 2.4048, the Bessel function changes sign and $J_{\text {eff }}$ becomes negative. Accordingly, if we view the driving field as acting simply to renormalize the tunneling, the product $\left(J_{\text {eff }} g\right)$ now becomes negative and dynamical instability can indeed occur. A similar effect would occur in an undriven system if $g$ were made negative (for example, by using a Feshbach resonance), for which the condensate would become attractive and thus unstable toward collapse. When $J_{\text {eff }}$ becomes zero at $K / \omega=5.52$ we can see that $g_{c}$ again peaks due to CDT. The same pattern of behavior then repeats. Figure 1(b) also shows that $g_{c}$ scales quite accurately as $g_{c} \sim \omega^{2}$. Surprisingly, the zone of stability thus becomes wider at high driving frequencies although the acceleration of the lattice is much larger. A similar feature was seen in the analysis of an accelerated condensate [11], which found an increasing propensity to dynamical instability in the limit of low acceleration.

It is interesting to compare these results with recent experiments [4], which saw no evidence for dynamical instability. To do this it is first necessary to estimate the interaction parameter $g$. The experiment was performed in the

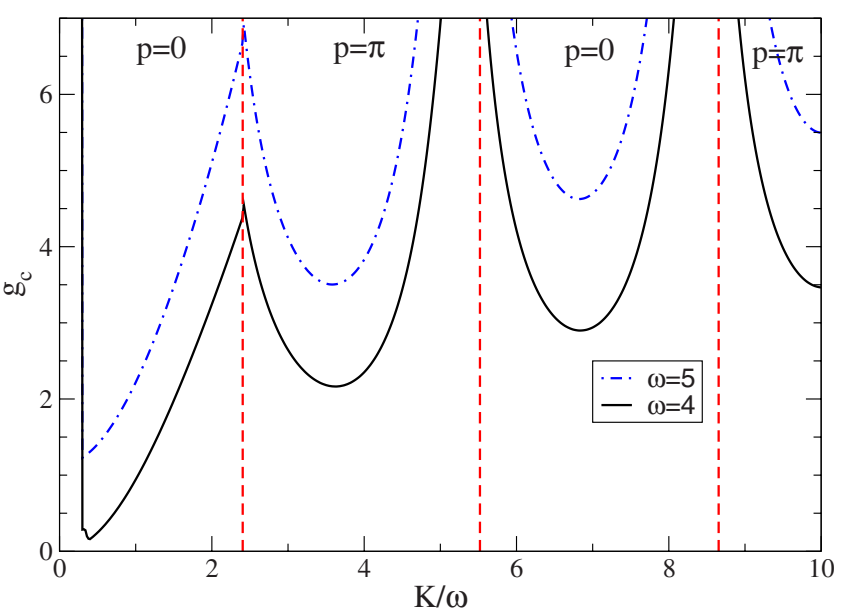

FIG. 2. (Color online) Critical interaction, $g_{c}$, for a system driven at frequencies of $\omega=4$ (solid black line) and $\omega=5$ (dotteddashed blue line). The Bessel function zeros are marked by the vertical dashed (red) lines. The labels $p=0$ and $p=\pi$ indicate the momentum of the Floquet state about which the instability analysis is made; when $J_{\text {eff }}$ is positive we use $p=0$, but when the effective tunneling is negative the analysis is done for $p=\pi$ (see text). For the higher driving frequency instability does not occur for $g \leq 1$, in agreement with experimental results [4].

mean-field regime and involved rather low values of interactions (with estimated Bose-Hubbard parameters of $U / J$ $\simeq 0.1$ ). The comparison is further complicated by the nonhomogeneous particle density produced by the trap potential. To proceed we first calculate the chemical potential for the system in the absence of the optical lattice by means of the Thomas-Fermi approximation, which will work well when the number of trapped atoms is large. For a sample of $\sim 10^{4}$ atoms of ${ }^{87} \mathrm{Rb}$, this yields a value of $\mu=300 \mathrm{~Hz}$ for the experimental trap parameters. The interaction energy can then be estimated [18] as $g=2 \mu / 7$, giving the result $g$ $\simeq 90 \mathrm{~Hz}$. The experiment employed a number of different optical lattice depths, giving undriven tunneling rates ranging from $J=350 \mathrm{~Hz}$ down to $J=90 \mathrm{~Hz}$ for the deepest lattices. Consequently, as an order of magnitude estimate, we are most interested in interaction strengths within the range $0.1<g / J<1$.

We show the stability boundaries obtained for two driving frequencies in Fig. 2. Let us first concentrate on the region $0 \leq \omega<2.4048$ where $J_{\text {eff }}$ is positive. We can see that for the lower driving frequency, $\omega=4$, dynamical instability can occur even for very weak interactions $(g \simeq 0.15)$ if the driving strength is tuned to a value of $K / \omega=0.40$. However, merely increasing the driving frequency to $\omega=5$ raises the value of the critical interaction strength to $g_{c}=1.2$, clearly demonstrating how higher driving frequencies render the system more robust against instability. This exceeds the maximum interaction strength present in the experiment and so indicates that for frequencies of $\omega \geq 5$ no dynamical instability effects should arise. As the experiment typically used driving frequencies on the order of a few kilohertz, corresponding to $\omega \sim 10 \mathrm{~J}$, our analysis corroborates the experimental finding of the absence of dynamical instability.

When $K / \omega$ is increased beyond the first zero of the Bessel function $(K / \omega=2.4048), J_{\text {eff }}$ becomes negative. Experimen- 

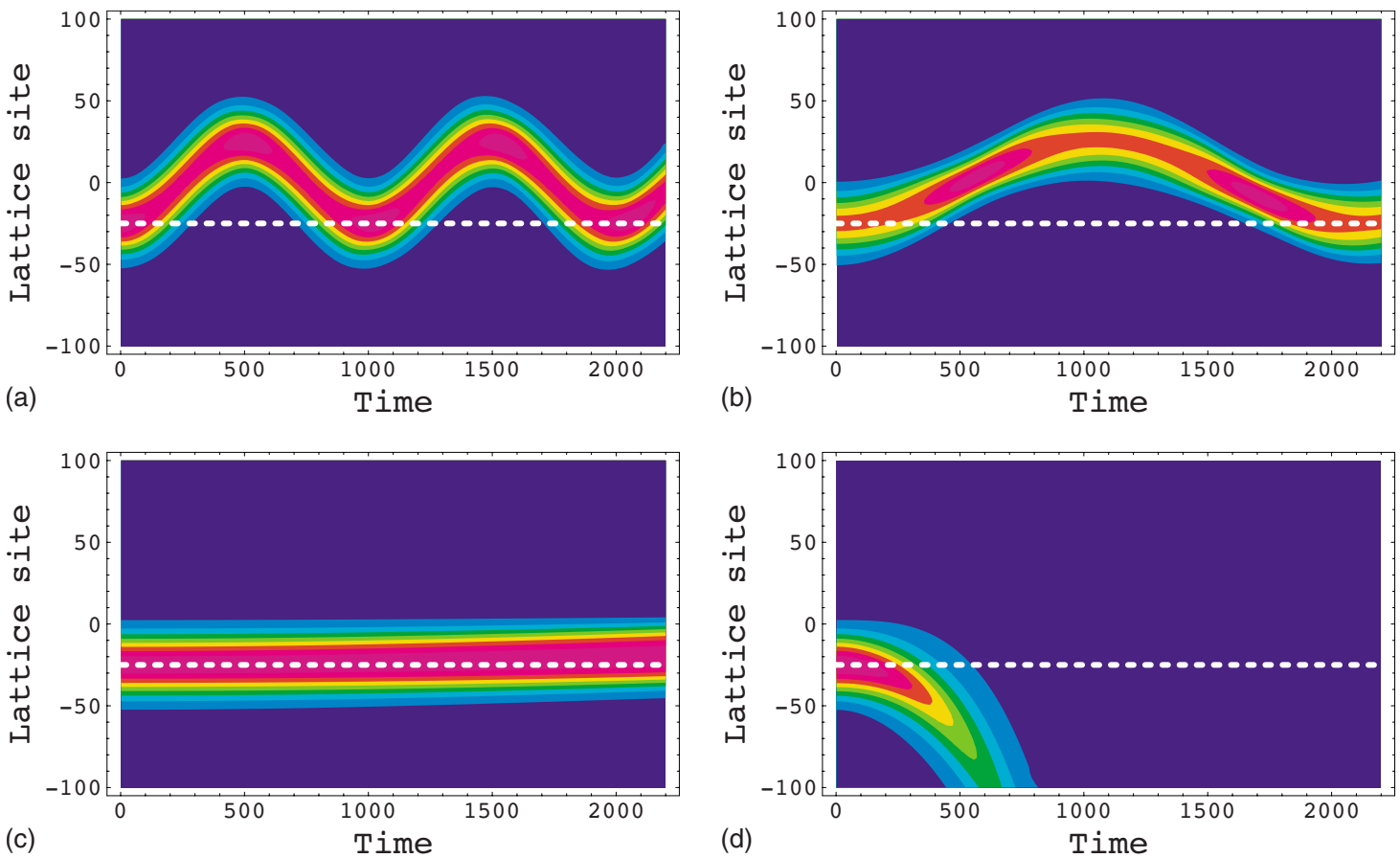

FIG. 3. (Color online) Dynamics of a condensate in a parabolic trap. Time is measured in units of the inverse tunneling $J^{-1}$. At $t=0$ the trap is shifted by 25 lattice spacings to induce the condensate into motion. System parameters are $g=0.1$ and $\omega=4$. (a) In the absence of driving, the condensate sloshes periodically from side to side of the trap with a well-defined frequency. (b) For $K / \omega=2, J_{\text {eff }}$ is reduced in amplitude, and so the frequency of the oscillation is correspondingly reduced. (c) For $K / \omega=2.40$, the tunneling is completely suppressed since $J_{\text {eff }} \simeq 0$, and the time evolution of the condensate is thus frozen. (d) For $K / \omega=3, J_{\text {eff }}$ becomes negative. The trap potential thus appears inverted to the condensate, which is rapidly expelled from the center.

tally it is seen that this causes the momentum distribution function to undergo a discrete shift, with the peaks now appearing at $p= \pm \pi$ instead of at $p=0$. This occurs [19] because the tunneling, as well as being renormalized in amplitude, acquires a phase factor of $\exp [i \pi]$. Consequently, as the initial state of the system is no longer of zero momentum, it is necessary to repeat the dynamical instability analysis around the Floquet state with $p=\pi$ to analyze the experimental results in this regime. This can be straightforwardly achieved by making the substitution $J \rightarrow-J$ in Eq. (8). From Fig. 2 we can clearly see that after peaking at $K / \omega=2.4048$, the value of $g_{c}$ then rapidly drops again, passing through a local minimum, before again peaking at the next zero of the Bessel function at $K / \omega=5.52$. This pattern of behavior then repeats, with sharp spikes in stability centered on the zeros of $\mathcal{J}_{0}$ where $J_{\text {eff }}$ vanishes, corresponding to CDT quenching the dynamics of the system.

We have so far considered an ideal flat optical lattice potential. In experiment, however, an additional harmonic trap potential is usually present which can substantially modify the dynamics of the system. To investigate this, we now apply an additional quadratic potential $V=k r_{i}^{2}$, where $r_{i}$ is measured from the center of the system. We initialize the system in the ground state of the mean-field Hamiltonian (2) in the presence of the trap and then displace the trapping potential by a distance of 25 lattice spacings, thereby exciting the condensate into motion.

In Fig. 3(a), we show the evolution of the condensate, in the absence of the periodic driving, in a shallow trap with a curvature of $k=0.00001$. The condensate makes a periodic oscillation of constant amplitude, very similar to the center of mass motion [8] observed in experiment. The period of oscillation is governed by the intersite tunneling or, equivalently, by the condensate's effective mass $\left(m^{*} \propto J_{\text {eff }}^{-1}\right)$. In Fig. 3(b) the system is subjected to a driving with $K / \omega=2$. Clearly the oscillation period has increased, corresponding to the expected reduction in the tunneling by $\mathcal{J}_{0}$, which can alternatively be interpreted as an enhancement of the effective mass. Increasing $K / \omega$ further to the first zero of $\mathcal{J}_{0}$ produces CDT, and so the system remains frozen in its initial state [Fig. 3(c)]. In this case the effective mass has become infinite.

A further increase in $K / \omega$ means that the Bessel function changes sign, and thus $J_{\text {eff }}$ becomes negative. We have seen already that this sign change has a significant effect on the dynamical stability of the ground state, and as we show in Fig. 3(d) it has an equally dramatic effect on the dynamics of the condensate. Instead of oscillating, the condensate now rapidly accelerates away from the center of the trap. The reason for this becomes evident when we examine the terms of the Hamiltonian. Making $J_{\text {eff }}$ negative is clearly equivalent to time-reversed evolution with a positive tunneling, but with reversed signs for the trapping potential and nonlinearity $g$. Thus when $J_{\text {eff }}$ changes sign, the condensate behaves as an attractive condensate in an inverted potential and so is quickly expelled from the center of the trap. It is interesting to note that the presence of the optical lattice imposes a fundamental spatial discretization on the system, and thus the wave packet's motion will in fact consist of a Bloch-type oscillation. As a consequence the wave packet will eventu- 
ally return to the center of the trap after being reflected from the band edge, which is a lattice-based effect not present in truly continuous systems.

\section{CONCLUSIONS}

In summary, we have analyzed how an oscillating driving potential can renormalize the effective tunneling, $J_{\text {eff }}$, of a Bose-Einstein condensate and have derived the Bogoliubov equations for this system. The stability of the condensate is sharply enhanced where $J_{\text {eff }}$ vanishes and, conversely, is least stable for driving parameters approximately midway between these points. The stability also depends strongly on the driving frequency, with high frequencies acting to suppress the onset of dynamical instability. This is particularly encouraging for the design of experiments since in the highfrequency regime dynamical instability can be completely avoided unless the interaction strength is extremely high. We have also shown how manipulating $J_{\text {eff }}$ in this way may also be used as a novel tool to control the dynamics of a condensate in a trap potential by tuning its effective mass to be positive, negative, or infinite. As this is achieved by controlling the amplitude of the tunneling, it is thus complementary to the well-known method of using Feshbach resonances to control the interaction. These both extend the possibility of manipulating the condensate to systems which do not possess convenient resonances and provide a means to investigate the interplay between nonlinearity and dispersion. A particularly exciting application of this technique would be to produce bright solitons in repulsive condensates [20], without using phase-imprinting techniques, by using a negative value of $J_{\text {eff }}$ to balance the interparticle repulsion.

\section{ACKNOWLEDGMENT}

The author was supported by the Ramón y Cajal program of the Spanish MCINN.
[1] F. Grossmann, T. Dittrich, P. Jung, and P. Hänggi, Phys. Rev. Lett. 67, 516 (1991).

[2] D. H. Dunlap and V. M. Kenkre, Phys. Rev. B 34, 3625 (1986).

[3] A. Eckardt, C. Weiss, and M. Holthaus, Phys. Rev. Lett. 95, 260404 (2005); C. E. Creffield and T. S. Monteiro, ibid. 96, 210403 (2006).

[4] H. Lignier, C. Sias, D. Ciampini, Y. Singh, A. Zenesini, O. Morsch, and E. Arimondo, Phys. Rev. Lett. 99, 220403 (2007).

[5] E. Kierig, U. Schnorrberger, A. Schietinger, J. Tomkovic, and M. K. Oberthaler, Phys. Rev. Lett. 100, 190405 (2008).

[6] C. E. Creffield, Phys. Rev. Lett. 99, 110501 (2007).

[7] B. Wu and Q. Niu, Phys. Rev. A 64, 061603(R) (2001).

[8] S. Burger, F. S. Cataliotti, C. Fort, F. Minardi, M. Inguscio, M. L. Chiofalo, and M. P. Tosi, Phys. Rev. Lett. 86, 4447 (2001).

[9] L. Fallani, L. De Sarlo, J. E. Lye, M. Modugno, R. Saers, C. Fort, and M. Inguscio, Phys. Rev. Lett. 93, 140406 (2004).

[10] M. Cristiani, O. Morsch, N. Malossi, M. Jona-Lasinio, M. Anderlini, E. Courtade, and E. Arimondo, Opt. Express 12, 4
(2004).

[11] Y. Zheng, M. Kŏstrun, and J. Javanainen, Phys. Rev. Lett. 93, 230401 (2004).

[12] B. Eiermann, P. Treutlein, Th. Anker, M. Albiez, M. Taglieber, K.-P. Marzlin, and M. K. Oberthaler, Phys. Rev. Lett. 91, 060402 (2003); L. Fallani, F. S. Cataliotti, J. Catani, C. Fort, M. Modugno, M. Zawada, and M. Inguscio, ibid. 91, 240405 (2003).

[13] D. Jaksch, C. Bruder, J. I. Cirac, C. W. Gardiner, and P. Zoller, Phys. Rev. Lett. 81, 3108 (1998).

[14] O. Morsch, J. H. Müller, M. Cristiani, D. Ciampini, and E. Arimondo, Phys. Rev. Lett. 87, 140402 (2001).

[15] M. Holthaus, Phys. Rev. Lett. 69, 351 (1992).

[16] Y. Castin and R. Dum, Phys. Rev. Lett. 79, 3553 (1997).

[17] B. Wu and Q. Niu, New J. Phys. 5, 104 (2003).

[18] C. J. Pethick and H. Smith, Bose-Einstein Condensation in Dilute Gases (Cambridge University Press, Cambridge, 2002).

[19] C. E. Creffield and F. Sols, Phys. Rev. Lett. 100, 250402 (2008).

[20] C. E. Creffield (unpublished). 